\title{
Unterschiede in der Selbst- und Fremdbeurteilung gesundheits- bezogener Lebensqualität bei Patienten mit leichter kognitiver Beeinträchtigung und Demenz vom Alzheimer-Typ \\ Differences Between Self- and Proxy-Assessment of Health- Related Quality of Life in Patients with Mild Cognitive Impairment and Alzheimer's Disease
}

\author{
Autoren \\ Philipp Heßmann,2, Maren Dreier ${ }^{3}$, Iris Brandes ${ }^{3}$, Richard Dodel' ${ }^{1}$, Erika Baum4, Matthias J. Müller ${ }^{5}$, \\ Monika Balzer-Geldsetzer ${ }^{1}$
}

Institute

1 Klinik für Neurologie, Philipps-Universität Marburg

2 Klinik für Psychiatrie und Psychotherapie, Universitätsmedizin Göttingen

3 Institut für Epidemiologie, Sozialmedizin und Gesundheitssystemforschung, Medizinische Hochschule Hannover

4 Abteilung für Allgemeinmedizin, Präventive und Rehabilitative Medizin, Philipps-Universität Marburg

5 Vitos Klinikum für Psychiatrie und Psychotherapie Gießen-Marburg und Justus-Liebig-Universität Gießen

Schlüsselwörter

gesundheitsbezogene Lebensqualität, Demenz vom Alzheimer-Typ, Selbst- und Fremdbeurteilung

Keywords

Alzheimer's disease, quality of life, self-assessment, proxy bias

Bibliografie

DOI http://dx.doi.org/10.1055/s-0042-121169

Online-Publikation: 27.2.2017 | Psychiat Prax 2018; 45:

78-86

(c) Georg Thieme Verlag KG Stuttgart · New York

ISSN 0303-4259

Korrespondenzadresse

Prof. Dr. med. Richard Dodel, MPH, Klinik für Neurologie, Philipps-Universität Marburg, Baldingerstraße, 35043

Marburg

dodel@med.uni-marburg.de

\section{ZUSAMMENFASSUNG}

Ziel Patienten mit einer Alzheimer-Demenz (AD) und leichten kognitiven Beeinträchtigungen (LKB) beurteilen ihre gesundheitsbezogene Lebensqualität (LQ) häufig besser als ihre Angehörigen. Einflussfaktoren dieses Beurteilungsunterschieds werden evaluiert.

Methodik Die LQ von 241 zu Hause lebenden Patienten wurde anhand des krankheitsspezifischen Quality of LifeAlzheimer's Disease-Fragebogens (QoL-AD) erhoben.

Ergebnisse Das Verwandtschaftsverhältnis, depressive Symptome und die Alltagsfunktionalität stellen relevante Einflussfaktoren bei Beurteilungsunterschieden der LQ dar. Schlussfolgerung Soziodemografische und klinische Variablen können Bewertungsunterschiede der LQ von Patienten mit AD und LKB teilweise erklären.

\section{ABSTRACT}

Objective The self-assessment of health-related quality of life (HrQoL) in patients with Alzheimer's disease (AD) and mild cognitive impairment is commonly higher than the proxy-assessment by caregivers. This study aims at evaluating sociodemographic and clinical factors to explain this difference.

Methods HrQoL of 241 community-dwelling patients was analysed using the dementia-specific Quality of Life-Alzheimer's Disease questionnaire (QoL-AD). Behavioural and psychological symptoms and functional capacity were evaluated using the Geriatric Depression Scale (GDS), the Neuropsychiatric Inventory (NPI) and the Alzheimer's Disease Cooperative Study-Activities of Daily Living scale (ADCSADL).

Results The self-assessment of patients' HrQoL was significantly higher than the caregiver-ratings (mean difference: $7.4 \pm 5.6, p<0.001$ ). Considerable influencing factors were the extent of depressive symptoms (GDS), the degree of impairment in functional performance (ADCS-ADL) and the relationship between patients and caregivers.

Conclusion Independent variables explained $23 \%$ of the variance in the difference between self- and proxy-assessment of HrQoL. Future studies should include further influencing factors such as caregivers' mental health. 


\section{Einleitung}

In Deutschland zählt die Demenz vom Alzheimer-Typ (AD) mit über 600000 Patienten zu den häufigsten Erkrankungen des höheren Lebensalters, wobei zukünftig von einer weiteren Zunahme der Betroffenen auszugehen ist [1]. Die AD führt zu einer progredienten Abnahme der kognitiven, physischen und alltäglichen Funktionsfähigkeit. Die Patienten leiden zudem häufig unter psychischen und Verhaltensstörungen, wie z.B. Depressionen [2]. Gegenwärtig sind keine effektiven präventiven oder kurativen Behandlungsansätze verfügbar. Daher sind die Reduktion von Inzidenz und Mortalität überwiegend ungeeignete Zielgrößen medizinischer Interventionen. Stattdessen stellt die Verbesserung der gesundheitsbezogenen Lebensqualität (LQ) ein zentrales, patientenrelevantes Versorgungsziel von Personen mit AD und leichter kognitiver Beeinträchtigung (LKB) dar. Vor diesem Hintergrund ist eine möglichst valide und reliable Erfassung der LQ erforderlich [3].

Die LQ beschreibt ein komplexes, multidimensionales Konstrukt, das physische, psychische und funktionale Aspekte umfasst und der subjektiven Bewertung unterliegt [4]. Aufgrund der kognitiven Defizite erfolgt bei Patienten mit AD und LKB neben der Eigenbeurteilung häufig ergänzend oder ersatzweise eine Fremdbeurteilung der LQ. Aus früheren Studien ist bekannt, dass Angehörige die LQ der Patienten häufiger schlechter beurteilen als die Patienten selbst $[5,6]$. Als relevante Einflussfaktoren der Bewertungsunterschiede wurden dabei u.a. eine reduzierte Krankheitseinsicht und Alltagsfunktionalität sowie psychische und Verhaltensstörungen beschrieben. Auch eine hohe Belastung und psychische Erkrankungen der Angehörigen waren mit dem Beurteilungsunterschied assoziiert [5 - 7]. Da die LQ bei Patienten mit AD und LKB ein wichtiges Ziel der gesundheitlichen Versorgung darstellt und klinische bzw. gesundheitspolitische Entscheidungen an diesem Ziel ausgerichtet sind, ist eine Analyse der Diskrepanz zwischen Eigen- und Fremdbeurteilung notwendig.

In Deutschland wurden bisher kaum Studien durchgeführt, in denen Unterschiede hinsichtlich der Eigen- und Fremdbeurteilung der LQ von Patienten mit AD und LKB analysiert wurden $[8,9]$. Es soll daher geprüft werden, ob soziodemografische Parameter der Patienten und ihrer Angehörigen sowie klinische Faktoren (Kognition, Alltagsfunktionalität, psychische und Verhaltensstörungen) mit dem Beurteilungsunterschied assoziiert sind.

\section{Methoden}

\section{Studiendesign}

In einer multizentrischen Querschnittstudie wurde die Versorgungssituation von insgesamt 395 zu Hause $(n=272)$ und in Pflegeeinrichtungen $(n=123)$ lebenden Patienten mit LKB und AD sämtlicher Schweregrade evaluiert. Für diese Publikation werden ausschließlich zu Hause lebende Patienten berücksichtigt, die ihre LQ höher bewerteten als ihre nächsten Angehörigen $(n=241)$.

Die Datenerhebung wurde von Februar 2009 bis Juli 2010 im Landkreis Marburg-Biedenkopf und Gießen durchgeführt
(Ethikkommission der Philipps-Universität Marburg: AZ 46/08 und Ethikkommission der Landesärztekammer Hessen: MC 29/ 2009). Die Rekrutierung erfolgte konsekutiv in folgenden Studienzentren: Vitos Klinik für Psychiatrie und Psychotherapie in Marburg, niedergelassene Hausärzte und Neurologen im Raum Marburg-Biedenkopf und Gießen sowie Gedächtnisambulanz der Philipps-Universität Marburg. Nach den Kriterien des National Institute of Neurological and Communicative Disorders and Stroke and Alzheimer's Disease and Related Disorders Association (NINCDS-ADRDA) wurden Patienten mit einer klinisch wahrscheinlichen AD oder LKB eingeschlossen [10]. Ausgeschlossen wurden Patienten mit anderen primären oder sekundären Demenzformen. Für eine detaillierte Beschreibung des Studiendesigns wird auf 2 frühere Publikationen verwiesen $[11,12]$.

\section{Gesundheitsbezogene Lebensqualität - QoL-AD}

Zur krankheitsspezifischen Eigen- und Fremdbeurteilung der LQ wurde der Quality of Life-Alzheimer's Disease Fragebogen (QoL-AD) eingesetzt [13]. Dabei werden 13 Items auf einer 4stufigen Likert-Skala (1=schlecht; 2 =zufriedenstellend; 3 = gut; 4 = ausgezeichnet) beurteilt, woraus sich ein Gesamtwert von minimal 13 und maximal 52 Punkten ergibt. Die LQ wird umso besser beurteilt, je höher der Punktwert ist. Bei maximal 2 nicht beantworteten Items wird ein korrigierter Summenwert gebildet. Der QoL-AD ist ein valides und reliables Testinstrument für verschiedene Schweregrade der Demenz [14]. Für jedes Paar aus Patient und nächstem Angehörigen wurde der Beurteilungsunterschied im QoL-AD durch Subtraktion des Wertes der Angehörigenbeurteilung von dem der Eigenbeurteilung berechnet.

Der QoL-AD kann v.a. von Patienten mit leichter bis mittelschwerer Demenz valide beantwortet werden. Frühere Studien zeigten jedoch, dass der QoL-AD auch bei Patienten mit schwerer Demenz eine ausreichende Validität und Reliabilität aufweist [15]. Allerdings sind die Validität und Reliabilität des QoL-AD bei Patienten mit sehr niedrigen MMST-Werten nur unzureichend belegt [16]. Insbesondere Defizite des visuellen und auditiven Sprachverständnisses können die valide und reliable Datengewinnung negativ beeinflussen.

\section{Kognitive Leistungsfähigkeit - MMST}

Die Beurteilung der kognitiven Leistungsfähigkeit erfolgte mit dem Mini-Mental Status Test (MMST), der 30 Items umfasst [17]. Anhand der Punktwerte wurde eine Einteilung in folgende Schweregradgruppen der Demenz vorgenommen: a) 27-30 Punkte: leichte kognitive Beeinträchtigung, b) 20-26 Punkte: leichte Demenz, c) 10 - 19 Punkte: mittelschwere Demenz und d) 0 - 9 Punkte: schwere Demenz [18].

\section{Psychische und Verhaltensstörungen - GDS und NPI}

Das Vorliegen von depressiven Symptomen wurde anhand der Geriatrischen Depressionsskala (GDS) evaluiert, die von den Patienten beantwortet wurde [19]. Höhere Werte zeigen eine stärkere Ausprägung der Symptomatik an, ab 11 Punkten ist eine Depression wahrscheinlich (maximal 30 Punkte). Zur Fremdbeurteilung weiterer psychischer und Verhaltensstörun- 
> Tab. 1 Soziodemografische Charakteristika der Patienten unterteilt nach Geschlecht.

\begin{tabular}{|c|c|c|c|c|}
\hline & $\begin{array}{l}\text { gesamt } \\
\mathrm{n}(\%)\end{array}$ & $\begin{array}{l}\text { weiblich } \\
\text { n (\%) }\end{array}$ & $\begin{array}{l}\text { männlich } \\
\text { n (\%) }\end{array}$ & p-Wert* \\
\hline gesamt & $241(100)$ & $150(62,2)$ & $91(37,8)$ & \\
\hline Altersgruppen & & & & $p=0,208$ \\
\hline 46-60 Jahre & $12(5,0)$ & $5(3,3)$ & $7(7,7)$ & \\
\hline $61-70$ Jahre & $30(12,4)$ & $19(12,7)$ & $11(12,1)$ & \\
\hline 71 - 80 Jahre & $117(48,5)$ & $69(46,0)$ & $48(52,7)$ & \\
\hline 81 - 96 Jahre & $82(34,0)$ & $57(38,0)$ & $25(27,5)$ & \\
\hline Familienstand & & & & $\mathrm{p}<0,001$ \\
\hline Partnerschaft & $156(64,7)$ & $76(50,7)$ & $80(87,9)$ & \\
\hline keine Partnerschaft & $79(32,8)$ & $68(45,3)$ & $11(12,1)$ & \\
\hline Bildungsstand & & & & $p=0,010$ \\
\hline niedrig & $178(73,9)$ & $114(76,0)$ & $64(70,3)$ & \\
\hline höher & $54(22,4)$ & $27(18,0)$ & $27(29,7)$ & \\
\hline Wohnsituation & & & & $p=0,001$ \\
\hline allein lebend & $52(21,6)$ & $42(28,0)$ & $10(11,0)$ & \\
\hline nicht allein lebend & $180(74,7)$ & $99(66,0)$ & $81(89,0)$ & \\
\hline Pflegesituation & & & & $p=0,320$ \\
\hline keine Pflege & $115(47,7)$ & $66(44,0)$ & $49(53,8)$ & \\
\hline informelle Pflege & $91(37,8)$ & $60(40,0)$ & $31(34,1)$ & \\
\hline formelle Pflege & $35(14,5)$ & $24(16,0)$ & $11(12,1)$ & \\
\hline
\end{tabular}

gen wurde das Neuropsychiatrische Inventar (NPI) eingesetzt [2]. Im NPI werden Häufigkeit und Schwere von zehn psychischen Störungsbildern evaluiert. Daraus wird der NPI-Gesamtwert berechnet, der umso höher ausfällt, je häufiger und schwerwiegender die Symptome auftreten. Der NPI-Belastungswert gibt an, in welchem Ausmaß sich Angehörige durch die Symptomatik belastet fühlen.

\section{Alltagsfunktionalität - ADCS-ADL}

Die Alltagsfunktionalität der Patienten wurde anhand des Alzheimer's Disease Cooperative Study-Activities of Daily LivingFragebogens (ADCS-ADL) als Fremdbeurteilung erhoben [20]. Durch Addition der Punktwerte aller 23 Items können maximal 78 Punkte erreicht werden. Je höher der Gesamtwert ausfällt, desto besser ist die Alltagsfunktionalität der Patienten.

\section{Statistische Analysen}

Die statistische Auswertung erfolgte mit SPSS Version 22.0 für Windows (IBM SPSS Statistics, Armonk, USA) und Excel 2010 für Windows (Microsoft Corporation, Redmond, USA).

Die Ergebnisse werden als Minimum, Median, Maximum und Mittelwert mit Standardabweichung oder als absolute bzw. relative Häufigkeiten angegeben. Vor der Auswertung wurde ein
Signifikanzniveau $\alpha$ von 0,05 festgelegt. Als nicht parametrische Testverfahren wurden bei 2 unabhängigen Stichproben der Mann-Whitney-Test, bei 2 verbundenen Stichproben der Wilcoxon-Vorzeichen-Rangtest und bei mehr als 2 unverbundenen Stichproben der Kruskal-Wallis-Test eingesetzt. Parametrische Tests umfassten den t-Test für 2 verbundene oder unverbundene Stichproben bzw. die einfaktorielle Varianzanalyse ( $F$ Test) für mehr als 2 unabhängige Stichproben. Bei Variablen mit nominalem Skalenniveau wird zur Überprüfung von Häufigkeitsunterschieden der Chi-Quadrat-Anpassungstest verwendet. Um die Stärke eines Zusammenhangs zwischen 2 metrischen Variablen zu quantifizieren, wird entweder der Korrelationskoeffizient nach Spearman $\left(r_{s}\right)$ oder der Pearson'sche Korrelationskoeffizient $(r)$ berechnet.

Um den Einfluss soziodemografischer und klinischer Parameter auf den Beurteilungsunterschied im QoL-AD zu analysieren, werden multiple lineare Regressionsmodelle eingesetzt. Voraussetzungen der linearen Regressionsanalyse (Ausschluss von Multikollinearität, Autokorrelation oder Heteroskedastizität) wurden im Vorfeld geprüft. 
- Tab. 2 Beurteilungsunterschiede im QoL-AD nach soziodemografischen Merkmalen der Patienten und Angehörigen.

\begin{tabular}{|c|c|c|c|c|c|c|}
\hline & \multicolumn{6}{|c|}{ Beurteilungsunterschied im QoL-AD } \\
\hline & n (\%) & $M W \pm S D$ & Min & Med & Max & p-Wert* \\
\hline Pflegesituation (Patienten) & & & & & & $p=0,008$ \\
\hline keine Pflege & $115(47,7)$ & $6,8 \pm 4,9$ & 1 & 6,0 & 18 & \\
\hline informelle Pflege & $91(37,8)$ & $7,0 \pm 5,9$ & 1 & 5,0 & 22 & \\
\hline formelle Pflege & $35(14,5)$ & $10,4 \pm 6,4$ & 1 & 10,0 & 23 & \\
\hline Alter in Jahren (Angehörige) & & & & & & $p=0,027$ \\
\hline $30-50$ & $54(22,4)$ & $7,7 \pm 5,3$ & 1 & 6,5 & 20 & \\
\hline $51-60$ & $64(26,6)$ & $8,6 \pm 5,7$ & 1 & 7,5 & 22 & \\
\hline $61-70$ & $42(17,4)$ & $7,7 \pm 5,4$ & 1 & 7,0 & 23 & \\
\hline $71-80$ & $59(24,5)$ & $6,3 \pm 5,7$ & 1 & 5,0 & 22 & \\
\hline $81-87$ & $22(9,1)$ & $5,7 \pm 6,0$ & 1 & 3,0 & 20 & \\
\hline Beziehung zum Patienten & & & & & & $p<0,001$ \\
\hline (Schwieger-)Tochter & $68(28,2)$ & $7,6 \pm 5,8$ & 1 & 6,0 & 22 & \\
\hline (Schwieger-)Sohn & $26(10,8)$ & $8,1 \pm 5,6$ & 1 & 7,5 & 19 & \\
\hline Ehe-/Lebenspartner & $117(48,5)$ & $6,3 \pm 5,4$ & 1 & 5,0 & 23 & \\
\hline anderes Verhältnis & $30(12,4)$ & $10,8 \pm 4,9$ & 1 & 12,0 & 17 & \\
\hline berufliche Veränderungen (Angehörige) & & & & & & $p=0,041$ \\
\hline ja & $32(13,3)$ & $9,2 \pm 5,9$ & 1 & 9,5 & 22 & \\
\hline nein & $209(86,7)$ & $7,1 \pm 5,6$ & 1 & 6,0 & 23 & \\
\hline
\end{tabular}

\section{Ergebnisse}

\section{Soziodemografische Charakteristika der Teilnehmer}

Frauen waren im Patientenkollektiv häufiger vertreten als Männer. Die Hälfte aller Patienten war zwischen 71 und 80 Jahre alt. Die Mehrheit der Patienten (65\%; $n=156)$ lebte in einer festen Partnerschaft; 74\% ( $n=178)$ wiesen einen niedrigen Bildungsstand (kein Schulabschluss, Haupt- bzw. Volksschulabschluss) auf. Mehr als die Hälfte der Patienten ( $52 \%$; $n=126)$ nahm informelle Pflege durch Angehörige bzw. formelle Pflege durch Pflegedienste in Anspruch ( $>$ Tab. 1).

Die Mehrheit der Angehörigen ( $n=156 ; 65 \%)$ war weiblich. Insgesamt 117 Angehörige (49\%) waren Ehe- bzw. Lebenspartner der Patienten, 94 (39\%) waren (Schwieger-)Töchter bzw. (Schwieger-)Söhne der Patienten, 30 Angehörige (12\%) wiesen ein anderes Verhältnis (Neffe/Nichte, Enkelkind etc.) zum Patienten auf. Das Durchschnittsalter lag bei 62,1 $\pm 13,2$ Jahren. Die Mehrheit der Angehörigen ( $n=128 ; 53 \%)$ verfügte über einen höheren Bildungsabschluss (Abitur, Fachhochschul- oder Universitätsabschluss). Berufstätig waren 88 Angehörige (37\%), bei 32 (13\%) hatten berufliche Veränderungen aufgrund der Versorgung der Patienten stattgefunden (z. B. Reduktion der Arbeitszeit).

\section{Klinische Befunde der Patientenpopulation}

Bei 44 Teilnehmern (18\%) lag eine LKB vor, 108 (45\%) waren leicht, 66 Patienten (27\%) mittelschwer und 23 (10\%) schwer dement ( $\triangleright$ Tab. 2). Depressive Symptome wiesen 99 Patienten (41\%) auf (mittlerer GDS-Wert: 10,2 $\pm 5,5)$. Bei 94 Patienten (39\%) wurden Symptome einer Apathie beobachtet, jeweils 75 Patienten (31\%) zeigten gereizte oder aggressive Verhaltensweisen. Aggressives Verhalten wurde deutlich häufiger bei Männern als bei Frauen beobachtet (41 vs. $25 \%$, Chi-QuadratTest: $p=0,013)$. Der arithmetische Mittelwert für den ADCSADL lag bei 45,2 \pm 19,9 Punkten.

\section{Gesundheitsbezogene Lebensqualität - Beurteilungsunterschiede im QoL-AD}

Die Beurteilung der LQ durch die Patienten im QoL-AD lag bei durchschnittlich 32,5 \pm 5,9 Punkten. Die Fremdbeurteilung durch die Angehörigen betrug im Durchschnitt 25,1 \pm 6,3 Punkte. Damit war die Eigenbeurteilung der LQ durchschnittlich um 7,4 5,6 Punkte höher als die Fremdbeurteilung $(p<0,001)$. Der Beurteilungsunterschied im QoL-AD (im Folgenden QoL$\mathrm{AD}-\mathrm{BU})$ wies keine relevanten Zusammenhänge mit dem Geschlecht, Alter, Familien- und Bildungsstand oder der Wohnsituation (allein vs. nicht allein lebend) der Patienten auf. Der QoL-AD-BU war für Patienten, die auf formelle Pflegeleistungen 
- Tab. 3 Beurteilungsunterschiede im QoL-AD nach klinischen Merkmalen der Patienten.

\begin{tabular}{|c|c|c|c|c|c|c|}
\hline & \multicolumn{6}{|c|}{ Beurteilungsunterschied im QoL-AD } \\
\hline & $\mathrm{n}(\%)$ & $M W \pm S D$ & Min & Med & Max & p-Wert* \\
\hline Demenzschweregrad & & & & & & $p=0,127$ \\
\hline MMST 27-30 & $44(18,3)$ & $6,5 \pm 5,0$ & 1 & 5,0 & 17 & \\
\hline MMST $20-26$ & $108(44,8)$ & $6,8 \pm 5,1$ & 1 & 6,0 & 22 & \\
\hline MMST $10-19$ & $66(27,4)$ & $7,8 \pm 5,6$ & 1 & 6,0 & 20 & \\
\hline MMST $0-9$ & $23(9,5)$ & $10,8 \pm 8,0$ & 1 & 8,0 & 23 & \\
\hline depressive Symptome & & & & & & $p=0,006$ \\
\hline ja (GDS $11-30)$ & $99(41,1)$ & $5,9 \pm 4,4$ & 1 & 5,0 & 22 & \\
\hline nein (GDS $0-10)$ & $142(58,9)$ & $8,0 \pm 5,8$ & 1 & 7,0 & 22 & \\
\hline Wahnvorstellungen (NPI) & & & & & & $p=0,017$ \\
\hline ja & $75(31,1)$ & $9,6 \pm 6,5$ & 1 & 9,0 & 22 & \\
\hline nein & $166(68,9)$ & $7,0 \pm 5,4$ & 1 & 6,0 & 23 & \\
\hline Aggressivität (NPI) & & & & & & $p=0,022$ \\
\hline ja & $75(31,1)$ & $8,6 \pm 6,0$ & 1 & 9,0 & 23 & \\
\hline nein & $166(68,9)$ & $6,9 \pm 5,4$ & 1 & 5,5 & 22 & \\
\hline Euphorie (NPI) & & & & & & $p=0,003$ \\
\hline ja & $22(9,1)$ & $10,9 \pm 5,9$ & 1 & 11,5 & 22 & \\
\hline nein & $219(90,9)$ & $7,1 \pm 5,5$ & 1 & 6,0 & 23 & \\
\hline Apathie (NPI) & & & & & & $p=0,001$ \\
\hline ja & $94(39,0)$ & $9,0 \pm 6,2$ & 1 & 8,0 & 23 & \\
\hline nein & $147(61,0)$ & $6,4 \pm 5,0$ & 1 & 5,0 & 22 & \\
\hline enthemmtes Verhalten (NPI) & & & & & & $p=0,043$ \\
\hline ja & $34(14,1)$ & $9,6 \pm 6,8$ & 1 & 9,0 & 23 & \\
\hline nein & $207(85,9)$ & $7,0 \pm 5,4$ & 1 & 6,0 & 22 & \\
\hline motorische Störungen (NPI) & & & & & & $\mathrm{p}<0,001$ \\
\hline ja & $46(19,1)$ & $10,3 \pm 6,0$ & 1 & 9,0 & 22 & \\
\hline nein & $195(80,9)$ & $6,7 \pm 5,3$ & 1 & 5,0 & 23 & \\
\hline
\end{tabular}

angewiesen waren, signifikant größer als für Personen, die keine oder informelle Pflege erhielten ( $>$ Tab. 2 ).

Für den QoL-AD-BU zeigten sich keine relevanten Assoziationen mit dem Geschlecht oder Bildungsstand der Angehörigen. Der QoL-AD-BU war jedoch bei jüngeren Angehörigen tendenziell größer als bei älteren. Der QoL-AD-BU war deutlich mit dem (Verwandtschafts-)Verhältnis zwischen Patienten und Angehörigen assoziiert und fiel bei Ehe- bzw. Lebenspartnern am geringsten aus. Die größte Differenz ergab sich für Angehörige, die weder Lebenspartner noch (Schwieger-)Kinder der Patienten waren. Der QoL-AD-BU war für Angehörige mit beruflichen Veränderungen infolge der Patientenversorgung deutlich größer als für jene, deren Beschäftigungssituation unverändert war ( $\triangleright$ Tab. 2).
- Tab. 3 zeigt, dass der QoL-AD-BU nicht signifikant mit dem Demenzschweregrad assoziiert war. Der QoL-AD-BU war geringer, wenn die Patienten depressive Symptome aufwiesen und nahm bei steigendem Schweregrad der depressiven Symptome gemäß GDS ab $\left(r_{s}=-0,210 ; p=0,001\right)$. Der QoL-AD-BU war jeweils größer, wenn die Patienten Wahnvorstellungen, aggressives oder enthemmtes Verhalten sowie euphorische oder apathische Zustände und psychomotorische Auffälligkeiten zeigten ( $\triangleright$ Tab.3). Der QoL-AD-BU war umso größer, je stärker die Ausprägung neuropsychiatrischer Symptome gemäß NPI-Gesamtwert war $\left(r_{s}=0,459 ; p<0,001\right)$. Ein vergleichbarer Zusammenhang konnte auch für den QoL-AD-BU und den NPI-Belastungswert gezeigt werden $\left(r_{s}=0,366 ; p<0,001\right)$. Außerdem fiel der QoL-AD-BU umso größer aus, je stärker die funktionalen 
- Tab. 4 Multiple lineare Regressionsmodelle mit dem Beurteilungsunterschied im QoL-AD als abhängige Variable und soziodemografischen Parametern der Patienten und Angehörigen (Modell 1 und Modell 2) sowie klinischen Parametern (Modell 3) als unabhängigen Variablen.

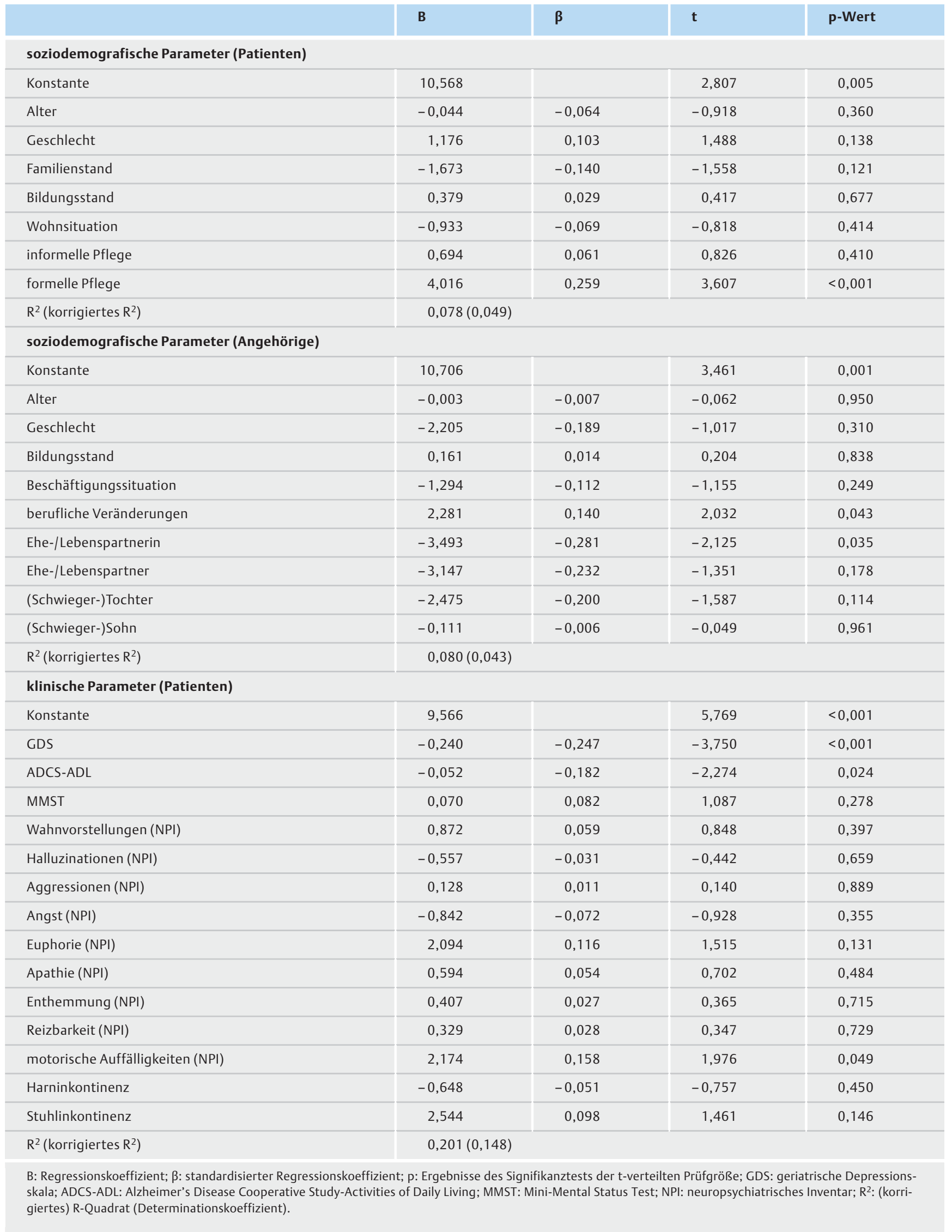


- Tab.5 Finales Modell der multiplen linearen Regressionsanalyse mit dem Beurteilungsunterschied im QoL-AD als abhängige Variable und ausgewählten soziodemografischen und klinischen Parametern als unabhängigen Variablen.

\begin{tabular}{|c|c|c|c|c|}
\hline & B & $\beta$ & $\mathbf{t}$ & p-Wert \\
\hline Konstante & 11,826 & & 5,482 & $<0,001$ \\
\hline \multicolumn{5}{|c|}{ soziodemografische Parameter (Patienten) } \\
\hline Geschlecht & 1,493 & 0,137 & 1,295 & 0,197 \\
\hline Familienstand & $-0,593$ & $-0,052$ & $-0,657$ & 0,546 \\
\hline formelle Pflege & 1,214 & 0,080 & 1,217 & 0,225 \\
\hline$R^{2}$ (korrigiertes $R^{2}$ ) & \multicolumn{4}{|c|}{$0,202(0,180)$} \\
\hline \multicolumn{5}{|c|}{ soziodemografische Parameter (Angehörige) } \\
\hline Geschlecht & $-0,335$ & $-0,030$ & $-0,214$ & 0,831 \\
\hline Beschäftigungssituation & $-1,846$ & $-0,169$ & $-1,899$ & 0,441 \\
\hline berufliche Veränderungen & 1,101 & 0,072 & 1,100 & 0,273 \\
\hline Ehe-/Lebenspartnerin & $-3,719$ & $-0,317$ & $-2,464$ & 0,015 \\
\hline Ehe-/Lebenspartner & $-3,834$ & $-0,297$ & $-2,906$ & 0,333 \\
\hline (Schwieger-)Tochter & $-1,573$ & $-0,134$ & $-1,056$ & 0,216 \\
\hline$R^{2}\left(\right.$ korrigiertes $\left.R^{2}\right)$ & \multicolumn{4}{|c|}{$0,229(0,196)$} \\
\hline \multicolumn{5}{|l|}{ klinische Parameter (Patienten) } \\
\hline GDS & $-0,230$ & $-0,240$ & $-3,920$ & $<0,001$ \\
\hline ADCS-ADL & $-0,043$ & $-0,154$ & $-2,050$ & 0,042 \\
\hline MMST & 0,066 & 0,078 & 1,03 & 0,300 \\
\hline motorische Auffälligkeiten (NPI) & 1,528 & 0,113 & 1,582 & 0,115 \\
\hline Euphorie (NPI) & 3,370 & 0,190 & 2,775 & 0,006 \\
\hline Stuhlinkontinenz & 2,937 & 0,115 & 1,803 & 0,073 \\
\hline $\mathrm{R}^{2}$ (korrigiertes $\mathrm{R}^{2}$ ) & \multicolumn{4}{|c|}{$0,282(0,230)$} \\
\hline
\end{tabular}

Beeinträchtigungen, gemessen anhand des ADCS-ADL-Gesamtwerts, waren $\left(r_{s}=-0,249 ; p<0,001\right)$.

- Tab. 4 zeigt die Ergebnisse von 3 Regressionsmodellen. Unter den soziodemografischen Merkmalen der Patienten hatte die Variable „Formelle Pflege“ den größten Einfluss auf den QoL-AD-BU. Bei Patienten, die durch ambulante Pflegedienste versorgt wurden, war der QoL-AD-BU demnach größer als bei Personen ohne Pflegebedarf (Referenzkategorie). Auch das (Verwandtschafts-)Verhältnis zwischen Patienten und Angehörigen wies eine starke Assoziation mit dem QoL-AD-BU auf (Referenzkategorie „anderes Verhältnis“ zu den Patienten). Insbesondere die Ausprägungen von depressiven Symptomen und funktionellen Einschränkungen erwiesen sich als relevante Einflussfaktoren des QoL-AD-BU. Motorische Auffälligkeiten und Euphorie erklärten ebenfalls einen hohen Anteil der Varianz.

Durch das finale Regressionsmodell konnten 23\% der Varianz erklärt werden ( $\triangleright$ Tab.5). Dabei wurden solche unabhängigen Variablen berücksichtigt, die in den vorherigen 3 Modellen relevante Zusammenhänge mit dem QoL-AD-BU zeigten. Unter allen Regressoren erklärte die Variable „Ehe-/Lebenspartner (in)“ der Patienten den größten Anteil der Varianz. Unter den klinischen Parametern hatten depressive Symptome bei den Patienten den größten Einfluss auf den QoL-AD-BU. Dabei war der QoL-AD-BU umso geringer, je ausgeprägter die depressiven Symptome waren. Der QoL-AD-BU war umso größer, je stärker die Alltagsfunktionalität der Patienten (ADCS-ADL) eingeschränkt war. Auch bei Vorliegen von „Euphorie“ war der QoLAD-BU signifikant größer als bei Patienten, die keine euphorischen Zustände zeigten.

\section{Diskussion}

Insbesondere bei chronischen oder letal verlaufenden Erkrankungen beurteilen Patienten ihre LQ häufig besser als ihre Angehörigen („disability paradox“) [21]. Dieses Phänomen sollte in der vorliegenden Arbeit bei Patienten mit AD und LKB untersucht werden. Daher wurden ausschließlich solche Pare aus Patienten und Angehörigen berücksichtigt, bei denen die Eigenbeurteilung durch die Patienten besser ausfiel als die Fremdbeurteilung durch Angehörige. Die subjektive LQ kann 
demnach trotz objektiv ungünstiger Bedingungen auch bei Patienten mit AD und LKB hoch sein. Mögliche Erklärungen dafür können eine Anpassung an die krankheitsbedingten Defizite sein, die zu einer Adaptation der eigenen Erwartungen, Präferenzen und Ansprüche führen [22]. Auch die Anpassung bestimmter Umgebungsfaktoren kann eine Rolle spielen (z. B. intensivierte Kontakte zu pflegenden Angehörigen der Demenzpatienten).

In der vorliegenden Studie konnte gezeigt werden, dass der Beurteilungsunterschied mit der (Verwandtschafts-)Beziehung zwischen Patienten und Angehörigen assoziiert war. Dabei war der Beurteilungsunterschied bei Ehe- und Lebenspartnern am geringsten. Möglicherweise entsteht bei Lebenspartnern mit engem Kontakt zum Patienten eine Adaptation an den krankheitsbedingten Zustand [7]. Die Versorgung der Patienten stellt für Lebenspartner zudem einen Teil des regulären Alltags dar, während andere Angehörige diese Aufgabe möglicherweise als größere Belastung empfinden [5].

Der Beurteilungsunterschied im QoL-AD war umso größer, je stärker die alltäglichen Fähigkeiten der Patienten eingeschränkt waren. Erklärt werden könnte dieser Zusammenhang durch eine reduzierte Wahrnehmung von Defiziten durch die Patienten bzw. eine Adaptation an das verminderte Kompetenzniveau [22]. Frühere Studien haben gezeigt, dass funktionale Einschränkungen einen stärker negativen Einfluss auf die Fremdals auf die Eigenbeurteilung haben [23]. Somit könnte eine stärkere Fokussierung der Angehörigen auf bestehende Defizite den Zusammenhang erklären. Es müsste jedoch eine Differenzierung der beeinträchtigten Domänen erfolgen. Betreffen die Einschränkungen z. B. intime Bereiche wie die Körperpflege, können diese wahrscheinlich auch die Eigenbeurteilung negativ beeinflussen.

Der Beurteilungsunterschied war zudem bei Patienten ohne depressive Symptome deutlich größer als bei Patienten mit depressiven Symptomen. Diese Ergebnisse sind mit denen früherer Studien vergleichbar [5-7]. Die wahrscheinlichste Erklärung dafür ist, dass sich bei Vorliegen depressiver Symptome die andernfalls hohe Eigenbewertung der LQ der Fremdbeurteilung durch die Angehörigen annähert. Die Definition einer depressiven Symptomatik und das Konstrukt der LQ stellen jedoch teilweise überlappende Konzepte dar [24]. Der QoL-AD greift u. a. mit den Items „Energie/Tatkraft“ und „Stimmungslage“ Aspekte auf, die von Personen mit depressiven Symptomen krankheitsbedingt schlechter beurteilt werden. Bei Vorliegen depressiver Symptome müsste demnach unterstellt werden, dass die LQ der Patienten grundsätzlich reduziert ist.

Die Fremdbeurteilung der LQ setzt voraus, stellvertretend für den Patienten eine Bewertung relevanter Lebensbereiche vorzunehmen. Dabei projizieren Angehörige auch ihre eigene Lebenssituation und psychische Verfassung auf die LQ der Patienten [25]. Demnach können Angehörige die LQ der Patienten unterschätzen, wenn sie selbst eine niedrige LQ aufweisen, unter psychischen oder körperlichen Beeinträchtigungen leiden oder sich durch die Pflege der Demenzpatienten belastet fühlen [5, 6]. Da diese Faktoren im Rahmen der Studie nicht abgefragt wurden, lässt sich evtl. auch die geringe Varianzaufklärung nachvollziehen. Aufgrund der Subjektivität des Konstrukts ist eine Verifizierung der Fremdbeurteilung nicht möglich. Die Fremdbeurteilung kann die Selbstbewertung ergänzen, aber nicht ersetzen [4]. Bei Demenzpatienten ist eine Eigenbeurteilung der LQ aufgrund der kognitiven Defizite jedoch häufig nicht mehr möglich. Daher besteht in klinischen und gesundheitspolitischen Entscheidungssituationen die Schwierigkeit festzulegen, ob primär die Eigen- oder die Fremdbeurteilung der LQ entscheidungsrelevant ist.

Bei der Eigen- und Fremdbeurteilung der LQ von Patienten mit $A D$ und $L K B$ sind vermutlich 2 verschiedene Perspektiven entscheidend [4]. Demnach orientieren sich Angehörige bei der Bewertung v.a. an funktionalen Aspekten und setzen die krankheitsbedingt eingeschränkten Fähigkeiten der Patienten in Relation zu einem früheren, höheren Kompetenzniveau. Demgegenüber ist die Bewertung der LQ durch die Patienten stärker vom gegenwärtigen affektiven Zustand abhängig. Für die Selbstbewertung der LQ sind vermutlich Faktoren wie die Zuwendung durch andere Menschen, das Gefühl von Sicherheit oder das Ausmaß körperlicher Beschwerden von größerer Bedeutung.

\section{Limitationen}

Die Datenerhebung fand in einem regional eng begrenzten Raum statt. Die Rekrutierung der Teilnehmer erfolgte konsekutiv und damit nicht zufallsbedingt. Daher sind generalisierbare Aussagen über die Grundgesamtheit aller Patienten mit AD und LKB in Deutschland nicht möglich. Einschränkend anzumerken ist zudem, dass bei der Analyse nicht zwischen Patienten mit $A D$ und Personen mit LKB unterschieden wurde. Personen mit LKB unterscheiden sich hinsichtlich des klinischen Erscheinungsbilds von $A D$-Patienten und entwickeln nicht zwangsläufig eine Demenz. Daher sollten diese beiden Populationen in zukünftigen Studien in Bezug auf Unterschiede in der Eigenund Fremdbeurteilung der LQ näher untersucht werden. Aufgrund des Querschnittdesigns der Studie können die berichteten Assoziationen zudem nicht als kausal angesehen werden. Durch den Einschluss solcher Personen, die im Studienzeitraum verfügbar und teilnahmebereit waren, besteht die Gefahr eines Selektionsbias. Bei Patienten mit AD und LKB besteht zudem die Möglichkeit einer fehlerhaften Erinnerung und einer Verzerrung der Ergebnisse im Sinne eines Reporting-Bias. Die Beurteilung der QoL-AD-Items basiert auf begrifflichen Unterschieden, für die eine Rangordnung und damit ordinales Skalenniveau festgelegt wird. In Anlehnung an frühere Studien wurde die Skalierung jedoch als quasi-metrisch aufgefasst, um eine quantitative Analyse des Beurteilungsunterschieds im QoL-AD zu ermöglichen [6]. Der QoL-AD beinhaltet auch Fragen zur kognitiven Leistungsfähigkeit und Alltagsfunktionalität. Da diese Fähigkeiten im Verlauf der Demenzerkrankung abnehmen, wird eine automatische Verschlechterung der LQ mit dem Erkrankungsprozess unterstellt. Aufgrund von Beeinträchtigungen des Sprachverständnisses und der kognitiven Defizite kann eine zuverlässige Datenerhebung anhand von Fragebögen zur LQ bei Patienten mit AD und LKB beeinträchtigt sein $[15,16]$. Es kann daher nicht sicher ausgeschlossen werden, dass die Studienergebnisse durch diese Defizite verzerrt wurden. 


\section{KONSEQUENZEN FÜR KLINIK UND PRAXIS}

- Die Verbesserung der LQ ist ein wichtiges Versorgungsziel von Patienten mit AD und LKB. Patienten beurteilen ihre LQ häufig höher als ihre Angehörigen. Dieser Unterschied sollte bei der Evaluation des Effekts medizinischer Maßnahmen auf die LQ in der alltäglichen Versorgung beachtet werden.

- Der Beurteilungsunterschied der LQ konnte v. a. durch das Ausmaß von depressiven Symptomen, Alltagseinschränkungen und das Verwandtschaftsverhältnis erklärt werden.

- Zukünftige Studien sollten weitere Einflussfaktoren (u.a. die psychische Verfassung der Angehörigen) untersuchen.

\section{Danksagung}

Die Studie wurde finanziell unterstützt durch die gemeinnützige Ernst und Margot Faber Stiftung in Marburg. Die Autoren bedanken sich bei allen beteiligten Patienten, Angehörigen und Kollegen für die Unterstützung bei der Studiendurchführung und -auswertung, insbesondere bei: PD Dr. Jens-Peter Reese, Dr. Greta Seeberg und Dr. Jürgen Rieke.

Interessenkonflikt

Die Autoren geben an, dass kein Interessenkonflikt besteht.

\section{Literatur}

[1] Ziegler U, Doblhammer G. Prevalence and incidence of dementia in Germany - a study based on data from the public sick funds in 2002. Gesundheitswesen 2009; 71: 281-290

[2] Cummings JL, Mega M, Gray K et al. The Neuropsychiatric Inventory: comprehensive assessment of psychopathology in dementia. Neurology 1994; 44: $2308-2314$

[3] Riepe MW, Mittendorf T, Forstl H et al. Quality of life as an outcome in Alzheimer's disease and other dementias - obstacles and goals. BMC Neurol 2009; 9: 47-55

[4] Gertz H], Berwig M. Critical observations on measuring quality of life of persons suffering from dementia. Nervenarzt 2008; 79: $1023-$ 1035

[5] Sands LP, Ferreira P, Stewart AL et al. What explains differences between dementia patients' and their caregivers' ratings of patients' quality of life? Am J Geriatr Psychiatry 2004; 12: 272 - 280

[6] Zucchella C, Bartolo M, Bernini S et al. Quality of life in Alzheimer disease: a comparison of patients' and caregivers' points of view. Alzheimer Dis Assoc Disord 2015; 29: 50 - 54

[7] Conde-Sala JL, Rene-Ramirez R, Turro-Garriga $O$ et al. Severity of dementia, anosognosia, and depression in relation to the quality of life of patients with Alzheimer disease: discrepancies between patients and caregivers. Am J Geriatr Psychiatry 2014; 22: 138-147
[8] Graske J, Meyer S, Wolf-Ostermann K. Quality of life ratings in dementia care - a cross-sectional study to identify factors associated with proxy-ratings. Health Qual Life Outcomes 2014; 12: 177-188

[9] Beerens HC, Sutcliffe C, Renom-Guiteras A et al. Quality of life and quality of care for people with dementia receiving long term institutional care or professional home care: the European RightTimePlaceCare study. J Am Med Dir Assoc 2014; 15: 54-61

[10] Blacker D, Albert MS, Bassett SS et al. The National Institute of Mental Health Genetics Initiative. Reliability and validity of NINCDS-ADRDA criteria for Alzheimer's disease. Arch Neurol 1994; 51: 1198 - 1204

[11] Reese JP, Hessmann P, Seeberg G et al. Cost and care of patients with Alzheimer's disease: clinical predictors in German health care settings. J Alzheimers Dis 2011; 27: 723-736

[12] Heßmann P, Seeberg G, Reese JP et al. Health-Related Quality of Life in patients with Alzheimer's disease in different German health care settings. J Alzheimers Dis 2016; 51: 545- 561

[13] Logsdon RG, Gibbons LE, McCurry SM et al. Quality of life in Alzheimer's disease: Patient and caregiver reports. Journal of Mental Health and Aging 1999; 5: $21-32$

[14] Thorgrimsen L, Selwood A, Spector A et al. Whose quality of life is it anyway? The validity and reliability of the Quality of Life-Alzheimer's Disease (QoL-AD) scale. Alzheimer Dis Assoc Disord 2003; 17: 201 208

[15] Logsdon RG, Gibbons LE, McCurry SM et al. Assessing quality of life in older adults with cognitive impairment. Psychosom Med 2002; 64: $510-519$

[16] Hoe J, Katona C, Roch B et al. Use of the QOL-AD for measuring quality of life in people with severe dementia - the LASER-AD study. Age Ageing 2005; 34: $130-135$

[17] Folstein MF, Folstein SE, McHugh PR. "Mini-mental state". A practical method for grading the cognitive state of patients for the clinician. J Psychiatr Res 1975; 12: 189-198

[18] Deuschl G, Maier W et al. S3-Leitlinie Demenzen. In: Leitlinien für Diagnostik und Therapie in der Neurologie. (25.1.2016). Deutsche Gesellschaft für Neurologie, Hrsg. Im Internet: www.dgn.org/ leitlinien (Stand: 21.5.2016)

[19] Yesavage JA, Brink TL, Rose TL et al. Development and validation of a geriatric depression screening scale: a preliminary report. J Psychiatr Res 1982; 17: 37-49

[20] Galasko D, Bennett D, Sano M et al. An inventory to assess activities of daily living for clinical trials in Alzheimer's disease. The Alzheimer's Disease Cooperative Study. Alzheimer Dis Assoc Disord 1997; 11: 33 39

[21] Drum CE, Horner-Johnson W, Krahn GL. Self-rated health and healthy days: examining the "disability paradox". Disabil Health J 2008; 1: 71 78

[22] Roick C, Hinz A, Gertz H]. Is quality of life in dementia patients validly estimable? Psychiat Prax 2007; 34: 108-116

[23] Yeaman PA, Kim DY, Alexander JL et al. Relationship of physical and functional independence and perceived quality of life of veteran patients with Alzheimer disease. Am J Hosp Palliat Care 2013; 30: $462-466$

[24] Bosboom PR, Alfonso H, Eaton J et al. Quality of life in Alzheimer's disease: different factors associated with complementary ratings by patients and family carers. Int Psychogeriatr 2012; 24: 708-721

[25] Arons AM, Krabbe PF, Scholzel-Dorenbos C] et al. Quality of life in dementia: a study on proxy bias. BMC Med Res Methodol 2013; 13: $110-118$ 